\section{Efficacy of Short-term Teriparatide for Hip Osteonecrosis}

\section{To the Editor:}

Osteonecrosis of the femoral head is the result of the lack or decrease of blood supply to the bone leading to bone and cartilage cellular death, fracture, and finally collapse of articular surface ${ }^{1}$. The pathogenesis is still unclear, but it seems that it is the result of a variety of traumatic and atraumatic factors such as genetic predisposition and metabolic factors. Risk factors are high-dosage glucocorticoids, alcohol abuse, venous stasis, adipocyte hypertrophy, alteration of circulating lipids, and other diseases that facilitate intravascular coagulation and thrombus formation ${ }^{2}$.

Nonsurgical treatment is limited and consists of physical therapy and rehabilitation, protected weight-bearing, hyperbaric therapy, and the use of nonsteroidal antiinflammatory drugs (NSAID), bisphosphonates, nifedipine, and lipid-lowering agents ${ }^{3}$. Unfortunately, these treatments have shown not a real efficacy, but only partial results on pain or delaying fracture and articular collapse ${ }^{4}$.

Teriparatide (TPT), a recombinant synthetic version of the human parathyroid hormone (PTH), is a bone anabolic agent that stimulates new bone formation by increasing osteoblast number, promoting osteogenic differentiation of bone mesenchymal stem cells and improving cell survival ${ }^{5,6}$. We report a case of atraumatic hip osteonecrosis successfully treated with short-term TPT.

A 42-year-old man was sent to our attention for hip osteonecrosis (Figure 1) unresponsive to nonsurgical treatment and awaiting surgery. The patient was unsuccessfully treated for several months with a combination of intravenous bisphosphonates, oral NSAID, and hyperbaric therapy in association with hydrokinesitherapy. At the time of the visit, the patient was unable to stand up without crutches and/or to maintain a seated position over prolonged periods. Persistent spontaneous pain and acute severe pain were present at movement, with a marked limitation of hip movements.

The patient started treatment with daily TPT $20 \mathrm{mcg}$ associated with oral calcium and cholecalciferol (1250 mg/400 IU). The hydrokinesitherapy was maintained to improve muscles and to recover hip function. After 2 months, pain was significantly reduced, with a gradual recovery of hip movement The patient abandoned 1 crutch and was able to move independently and to sit for long hours, thus he was also able to return to work. After 3 months, the patient completely abandoned the use of walking aids, but still complained about tenderness from sitting to standing; MRI showed a marked reduction of bone edema without double line sign or cortical collapse (Figure 2). TPT was discontinued and hydrokinesitherapy maintained.

To date, at 15 months of followup, pain from sitting to standing remains and the surgical option will be considered only in case of worsening of the symptoms due to secondary osteoarthritis.

The natural history of hip osteonecrosis is usually characterized by the collapse of the articular surface and degenerative changes leading to surgery. Conservative treatments are generally not successful, and surgical options vary depending on disease stage; none of the surgical options is considered a gold standard. The prevention of femoral head collapse and maintenance of the hip function is of paramount importance. Although it is possible to recover function with total hip arthroplasty, delaying surgery is important in the early stages and in young patients. To date, the nonsurgical treatments proposed to prevent disease progression such as bisphosphonate, anticoagulants, lipid-lowering agents, and vasodilators have had very limited success even in the early stage. The rationale for TPT use in osteonecrosis is the mechanism of action and the regulatory hormonal function of parathormone TPT has been shown to reach the maximum anabolic effect in the first months of treatment when the bone remodeling is promoted ${ }^{7}$. Other factors may be involved in the anabolic and regulating effect of TPT on bone ${ }^{8}$, such as the upregulation of growth factors (basic fibroblast growth factor- 2 and insulin-like growth factor-1, receptor activator of nuclear factor- $\kappa \mathrm{B}$ ligand), the influence on the Wingless/ $\beta$-catenin signaling pathway, and the transcriptional suppression of the sclerostin gene. Moreover, intermittent PTH

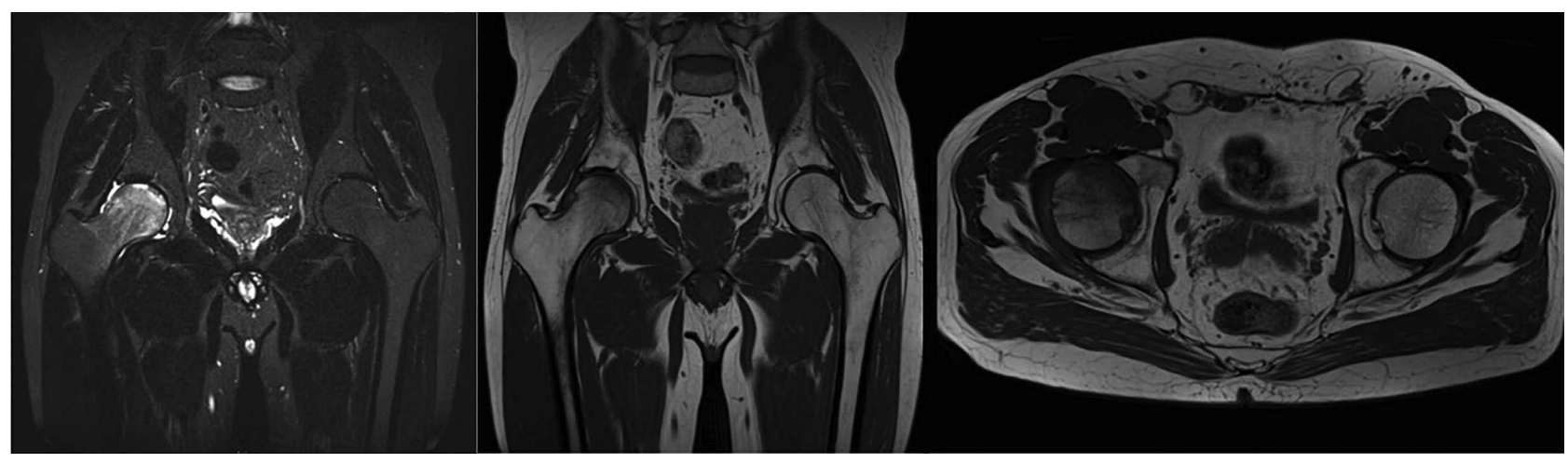

Figure 1. Magnetic resonance imaging before treatment.

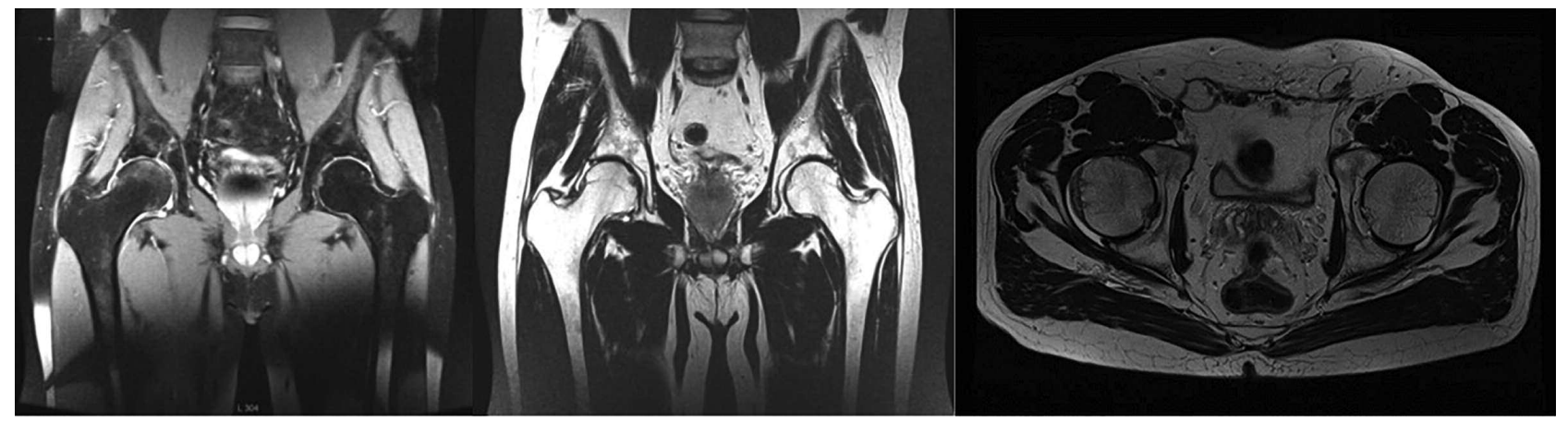

Figure 2. Magnetic resonance imaging after treatment.

Personal non-commercial use only. The Journal of Rheumatology Copyright @ 2016. All rights reserved. 
reverses bone hypovascularity induced by radiation in mice through neoangiogenesis ${ }^{9}$. It has also been shown that TPT accelerates fracture healing and has a potential effect in increasing bone volume within joints and inhibiting articular cartilage degeneration ${ }^{10}$.

The rapid response in terms of pain and recovery of hip function with sustained effect demonstrates that TPT may have a key role in patients with early stage hip osteonecrosis. Rapid response to treatment and the possibility to discontinue the therapy may make TPT a helpful drug in the early management of osteonecrosis. In our patient, the pain on standing is the expression of the joint damage that has not been corrected by treatment. Thus, TPT may be considered as an early treatment option in the prevention of evolution to femoral head collapse, and to delay or even avoid the surgical option, especially in young patients. Further studies are warranted to confirm this suggestion.

FELICE GALLUCCIO, MD, Department of Clinical and Experimental Medicine, Division of Rheumatology, Azienda Ospedaliero-Universitaria Careggi (AOUC), University of Florence; MARCO

MATUCCI-CERINIC, MD, Professor, Department of Clinical and Experimental Medicine, Division of Rheumatology, AOUC, University of Florence, Florence, Italy. Address correspondence to Dr. F. Galluccio, SOD Reumatologia, Viale Pieraccini 18, Villa Monnatessa, 50139

Florence, Italy. E-mail: felicegalluccio@gmail.com

\section{REFERENCES}

1. Kaushik A, Das A, Cui Q. Osteonecrosis of the femoral head: an update in year 2012. World J Orthop 2012;3:49-57.
2. Mont MA, Hungerford DS. Non-traumatic avascular necrosis of the femoral head. J Bone Joint Surg Am 1995;77:459-74.

3. Chang CC, Greenspan A, Gershwin ME. Osteonecrosis: current perspectives on pathogenesis and treatment. Semin Arthritis Rheum 1993;23:47-69.

4. Moya-Angeler J, Gianakos AL, Villa JC, Ni A, Lane JM. Current concepts on osteonecrosis of the femoral head. World J Orthop 2015;6:590-601.

5. Canalis E, Giustina A, Bilezikian JP. Mechanisms of anabolic therapies for osteoporosis. N Engl J Med 2007;357:905-16.

6. Jilka RL. Molecular and cellular mechanisms of the anabolic effect of intermittent PTH. Bone 2007;40:1434-46.

7. Dhillon RS, Schwarz EM. Teriparatide therapy as an adjuvant for tissue engineering and integration of biomaterials. J Mater Res 2011;4:1117-31.

8. Rosen CJ. The role of parathyroid hormone in the management of osteoporosis. Horm Res 2005;64 Suppl 2:81-5.

9. Kang SY, Deshpande SS, Donneys A, Rodriguez JJ, Nelson NS, Felice PA, et al. Parathyroid hormone reverses radiation induced hypovascularity in a murine model of distraction osteogenesis. Bone 2013;56:9-15.

10. Kakar S, Einhorn TA, Vora S, Miara LJ, Hon G, Wigner NA, et al. Enhanced chondrogenesis and Wnt signaling in PTH-treated fractures. J Bone Miner Res 2007;22:1903-12.

J Rheumatol 2016;43:11; doi:10.3899/jrheum.160190 